\title{
Anaesthesia outside the operating room
}

Pirjo H. Manninen MD FRCPC

The skills of the anaesthetist are requested more frequently in different locations of the hospital outside the operating room. With rapid developments in medicine, there has been a proliferation in the number of procedures performed by nonsurgical physicians. Procedures may be uncomfortable or have severe complications. The anaesthetist's role is important to the safety and comfort of the patient. Some of the procedures for which the anaesthetist may be requested are listed in the Table.

\section{General considerations}

The reluctance to provide anaesthesia in unfamiliar environments can be eliminated by careful planning and understanding of the patient, the procedure, the available equipment, and the hazards of the environment. '

\section{Patient}

The assessment of the patient is the same as for any preoperative patient undergoing anaesthesia in the operating room. Some procedures are routinely done under general anaesthesia. The reasons for the request of an anaesthetist to be present in other situations is usually the inability of the patient to cooperate, the discomfort of the procedure, or a medically unstable patient. Uncooperative patients usually require general anaesthesia, others a neurolept anaesthetic or mild sedation, good monitoring and reassurance.

\section{Procedure}

In order to provide adequate and appropriate anaesthesia and monitoring, the anaesthetist must know what the procedure entails, how it is performed, how painful it is, the duration, and the potential complications.

\section{Equipment}

Equipment required for general anaesthesia (anaesthetic machine, suction, and oxygen source) should be present or readily available. The standard requirements of monitoring during anaesthesia in the operating room also apply to these patients. Monitors for ECG, BP, temperature, oxygen saturation, and end-tidal carbon dioxide are needed. Drugs for general anaesthesia as well as for emergency resuscitation should be readily available. If anaesthesia drugs are stocked in a remote area, one needs to ensure that the drugs are not outdated. Anaesthesia equipment such as iv supplies, airways, laryngoscope,
TABLE Procedures outside the operating room

Electroconvulsive therapy (ECT)

\section{Cardiology}

Cardioversion

Angiography

Pacemaker insertion

Defibrillator - insertion and check

Angioplasty

Radialogy

Angiography

CT scan

Magnetic resonance imaging (MRI)

Endoscopic retrograde cholangiopancreatography (ERCP)

Myelograms

Percutaneous rhizotomy

Interventional neuroradiology

Radiation therapy

Lishotripsy

endotracheal tubes, stethoscope, humidifying and warming devices and nasal prongs may need to be taken to the location. Standard defibrillation and resuscitation equipment should be located nearby. Some centres have a "travelling cart" with all the appropriate supplies ready to go.

\section{Hazards of the environment}

Outside the operating room, the anaesthetist has to fit in amongst the equipment required for the procedure. There may be limited access to the patient, the possibility of radiation exposure, dark, cold rooms, uncomfortable tables for the patient and a hostile environment for electronic monitoring. Also the anaesthetist may be very much alone in this environment. There may be nobody else in the room who understands the complexities of anaesthesia. The anaesthetist needs to know where there is assistance in the event of an emergency. A postanaesthetic care unit is the ideal place for recovery of the patient. If this is far away, one needs to ensure that the patient is fully awake and stable prior to transport.

The actual techniques of anaesthesia will vary accord-

From the Department of Anacsthesia, University of Western Ontario, London, Ont. N6A 5A5. 
ing to the procedure, location and individual requirements of the patient. The anaesthetic management of some of the more common procedures performed outside the operating room will be discussed. Application of these principles will provide a framework on which to plan an anaesthetic at any location.

\section{Electroconvulsive therapy (ECT)}

Although ECT therapy remains controversial, it is still widely practiced. ${ }^{2-5}$ It is performed in specific treatment areas of psychiatric and general hospitals under general anaesthesia. The grand mal seizure produced by ECT results in widespread physiological changes. Cardiovascular changes include an initial parasympathetic stimulation which may result in bradycardia and hypotension. Within seconds, a sympathetic surge occurs with tachycardia, hypertension, and arrhythmias.

\section{Reasons for general anaesthesia}

(1) Retrograde amnesia occurs after a successfully induced seizure, but the use of muscle relaxants to prevent fractures created a need for hypnosis. (2) Anaesthesia will minimize the physiological sequelae. (3) The anxiety of repetitive ECT treatments is greatly decreased by general anaesthesia.

\section{Anaesthetic considerations}

The patient should have a complete pretreatment anaesthetic evaluation. Since the increase in blood pressure may result in cardiovascular or cerebral injury, adequate control of blood pressure in a hypertensive patient should be obtained prior to ECT therapy. Most patients will be receiving some psychotropic medication such as tricyclics, lithium or monoamine oxidase inhibitors. The practice of stopping MAO inhibitors two weeks before an anaesthetic has been questioned. ${ }^{6}$ Lithium may interact with barbiturates to prolong recovery time, and may prolong the action of succinylcholine and pancuronium. 7,8

The factors that influence the choice of anaesthetic agents are: (1) onset and recovery of anaesthesia should be rapid with minimal side effects, (2) the drugs should not affect the duration of the seizure, and (3) as the procedure is repetitive the agents must be acceptable for repeated use.

\section{Anticholinergics}

The routine use of preoperative atropine or glycopyrrolate to attenuate vagal stimulation of the heart and decrease oral secretions has been shown to have no benefit, but it should be readily available. ${ }^{9}$

\section{Induction agents}

The most common drugs used are methohexitone and thiopentone. Others, including diazepam, ketamine, etomidate and, more recently, propofol, are also used. Diazepam increases the threshold for electrically induced seizures. Propofol will attenuate the cardiovascular changes and also shorten recovery ${ }^{10}$ but may shorten the duration of the seizure and thus decrease its therapeutic value. ${ }^{11}$ The pain produced by the injection of methohexitone and propofol is decreased by use of $i v$ lidocaine. ${ }^{12}$

\section{Muscle relaxants}

Succinylcholine is the most popular agent. Muscle pain is not common after succinylcholine following ECT. ${ }^{13}$ Nondepolarizing agents have been used in patients where succinylcholine is contraindicated. ${ }^{14}$

Proper modification of drug dosages and timing of the shock requires cooperation between the anaesthetist and psychiatrist. Damage to teeth, tongue, eyes, extremities and skin may occur if not prevented by a mouth gag and protection by attending personnel. Modification of the sympathetic response to the ECT with sodium nitroprusside, hydralazine, clonidine or propranolol should be considered in patients with cardiovascular disease. ${ }^{15}$ Preoxygenation and manual ventilation with oxygen until spontaneous ventilation resumes may decrease the incidence of arrhythmias. ${ }^{16}$ An Ambu bag can be used. The patient should be recovered on his side and observed closely for $15 \mathrm{~min}$ after the seizure, as this is the high-risk period. ${ }^{2}$

\section{Cardioversion}

Direct current countershock is used to restore sinus rhythm in cardiac arrhythmias such as atrial fibrillation and flutter, supraventricular and ventricular tachycardia. ${ }^{17}$ The patient may have an acute onset with haemodynamic instability requiring urgent treatment or the problem may be chronic with failure to respond to drug therapy and thus require elective cardioversion. Cardioversion is usually performed in cardiac laboratories or in monitored units. Cardioversion requires a short anaesthetic to eliminate the awareness of the electrical shock. It is unnecessary to paralyze the patient as there are only regional muscle contractions. Anaesthetic agents used include thiopentone, methohexitone, nitrous oxide, halothane, diazepam, midazolam and more recently propofol. Thiopentone is most widely used. A benzodiazepine may be considered the drug of choice in poor risk patients and in emergency situations as it results in less cardiovascular depression. ${ }^{18}$ However, patients may have some recall of the event. Advantages of midazolam are its shorter duration of action and minimal pain on injection. ${ }^{19}$ Propofol results in rapid recovery. ${ }^{20}$ Appropriate timing of events by the anaesthetist and cardiologist is essential. The patient should be oxygenated and if respiratory 
depression occurs, manual ventilation performed. When more than one shock has to be delivered for successful cardioversion one needs to ensure that the patient remains anaesthetized.

\section{Radiological procedures}

There are many diagnostic and therapeutic procedures performed in the radiology suite such as endoscopic retrograde cholangio pancreatography, where the sedation and monitoring of the patient is usually done by the physician doing the procedure. Occasionally the anaesthetist is requested to care for restless, uncooperative or unstable patients.

\section{Magnetic resonance imaging (MRI)}

The difficulties of anaesthetizing a patient in the MRI suite are the problems of the magnetic field. ${ }^{21,22}$ (1) The MRI suite is usually in a remote isolated location. (2) The cylindrical large-bore magnet surrounds the body limiting access to the patient. (3) Ferromagnetic objects may be hurled toward the scanner creating a lethal projectile. (4) Large metal objects may interfere with the quality of the image. (5) Many electronic instruments may not function normally close to the magnet. (6) Implantable ferrous magnetic devices such as older cerebrovascular clips, surgical clips and pacemakers are hazardous.

\section{Equipment}

Ferrous material is present in most anaesthetic equipment such as ventilators, vaporizers, gas cylinders, laryngoscope batteries, stethoscopes, connectors, and intravenous poles. Most equipment can be modified or constructed out of non-ferrous alloys. The anaesthetic machine presents the greatest challenge. It may be bolted to a wall in the MRI room or be left outside the scanning room. Another solution is to design or modify an anaesthetic machine for the MRI suite alone. ${ }^{23}$ MRI compatible ventilators are available. ${ }^{24}$ If piped gases are not available, gas cylinders must be kept outside or be replaced with aluminium cylinders.

\section{Monitors}

Modifications are required. ${ }^{25}$ Long tubing is essential for all monitors and intravenous lines. A telemetric ECG with filters to decrease interference from the scanner, a Döppler, or a plastic oesophageal or precordial stethoscope is used for heart rate monitoring. Modified BP cuff tubing can be adapted to a noninvasive automatic machine. Ventilation is monitored by an apnoea alarm mattress, chest bellows, pulse oximetry and/or capnography. If an emergency situation occurs, the magnetic field can be discontinued.

\section{Anaesthetic management}

For MRI scanning, most patients need mild sedation or neurolept analgesia but a few will require general anaesthesia. Some anaesthetists advocate endotracheal intubation for all patients requiring general anaesthesia. ${ }^{22}$ Spontaneous ventilation may be safer in many situations, though the medical condition of the patient may necessitate mechanical ventilation (ICU patients). The preferred approach and drugs for anaesthesia will depend on the patient and the available equipment.

\section{Interventional neuroradiology}

Invasive neuroradiological procedures, such as the embolization or balloon occlusion of arterial venous malformations, vascular tumours, and intracranial aneurysms and fistulae carry considerable risk of potentially disastrous complications. These include a sudden bleed from a ruptured lesion, acute cerebral ischaemia, or technical problems such as the migration of the catheter or balloon. These problems may result in sudden compromise of the patient's neurological and/or cardiorespiratory system. The patient may require urgent airway management or even urgent craniotomy. The monitoring of the patient includes continuous assessment of the neurological system. The ideal anaesthetic is neurolept anaesthesia which will allow for communication with the patient. ${ }^{26}$

\section{Summary}

Providing anaesthesia to patients outside the operating room will be a satisfying experience if one adheres to the same standards of care as in the operating room and one has thoroughly planned in advance the management of the patient.

\section{References}

1 Hughes $C W$. Anesthesia outside of the operating room. Seminars in Ancsthesia 1990; IX(3): 190-6.

2 Rich CL, Ty Smish $N$. Anaesthesia for electroconvulsive therapy: a psychiatric viewpoint. Can Anaesth Soc J 1981; 28: 153-7.

3 Marks $R J$. Electroconvulsive therapy: physiological and anaesthetic consideration. Can Anaesth Soc J 1984; 31: 541-8.

4 Selvin BL. Electroconvulsive therapy - 1987. Anesthesiology $1987 ; 67: 367-85$.

5 Gaines GY, Rees DI. Electroconvulsive therapy and anesthetic considerations. Anesth Analg 1986; 65: 1345-56.

6 El-Ganzouri AR, Ivankovich AD, Braverman B, McCarthy $R$. Monoamine oxidase inhibitors: should they be discontinued preoperatively? Anesth Analg 1985; 64: 592-6. 
7 Jephcott G, Kerry RJ. Lithium: an anaesthetic risk. $\mathrm{Br} \mathrm{J}$ Anaesth 1974; 46: 389-90.

8 Hill GE, Wong $K C$, Hodges MR. Lithium carbonate and neuromuscular blocking agents. Anesthesiology 1977; 46: 122-6.

9 Wyant GM, MacDonald WB. The role of atropine in electroconvulsive therapy. Anaesth Intensive Care 1980; 8: 445-50.

10 Boey WK, Lai FO. Comparison of propofol and thiopentone as anaesthetic agents for electroconvulsive therapy. Anaesthesia 1990; 45: 623-8.

11 Simpson KH, Halsall PJ, Carr CME, Stewart KG. Propofol reduces seizure duration in patients having anaesthesia for electroconvulsive therapy. Br J Anaesth 1988; 61: 343-4.

12 Simpson KH, Halsall PJ, Sides CA, Keeler JF. Pain on injection of methohexitone. The use of lignocaine to modify pain on injection of methohexitone during anaesthesia for electroconvulsive therapy. Anaesthesia 1989; 44: 688-9.

13 McCleave DJ, Blakemore WB. Anaesthesia for electroconvulsive therapy. Anaesth Intensive Care 1975; 3: 250-6.

14 Stack $C G$, Abernethy $M H$, Thacker $M$. Atracurium for ECT in plasma cholinesterase deficiency. Br J Anaesth 1988; 60: 244-5.

15 Anton AH, UY DS, Redderson CL. Autonomic blockade and the cardiovascular and catecholamine response to electroshock. Anesth Analg 1977; 56: 46-54.

16 Lew JKL, Eastley RJ, Hanning CD. Oxygenation during electroconvulsive therapy. Anaesthesia 1986; 41: 1092-7.

17 DeSilva RA, Graboys TB, Podrid PJ, Lown B. Cardioversion and defibrillation. Am Heart J 1980; 100: 881-95.

18 Orko R. Anaesthesia for cardioversion: a comparison of diazepam, thiopentone and propanidid. $\mathrm{Br} \mathrm{J}$ Anaesth 1976; 48: 257- 62 .

19 Krichbaum DW, Hamid I. Midazolam sedation and amnesia in elective cardioversion. Clin Pharm 1988; 7; 423.

20 Valtonen $M$, Kanto J, Klossner J. Anaesthesia for cardioversion: a comparison of propofol and thiopentone. Can J Anaesth 1988; 35: 479-83.

21 Burk NS. Anesthesia for magnetic resonance imaging. Anesthesiology Clinics of North America 1989; 7 : 707-21.

22 Nixon C, Hirsch NP, Ormerod IEC, Johnson G. Nuclcar magnctic resonance. Anacsthesia 1986; 41: 131-7.

23 Karlik SJ, Heatherley $T$, Pavan $F$ et al. Patient anesthesia and monitoring at a 1.5-T MRI installation. Magn Reson Med 1988; 7: 210-21.

24 Rao CC, MCNiece WL, Emhardt $J$. Modification of an anesthesia machinc for use during magnetic resonance imaging. Anesthesiology 1988; 68: 640-1.
25 Roth JL, Nugent M, Gray JE et al. Patient monitoring during magnetic resonance imaging. Anesthesiology 1985; 62: 80-3.

$26 O^{\prime}$ Mahony BJ, Bolsin SNC. Anaesthesia for closed embolisation of cerebral arteriovenous malformations. Anaesth Intensive Care 1988; 16: 318-23. 


\section{Anesthésie à l'éxtérieur de la salle d'opération}

Pirjo H. Manninen MD

L'expertise de l'anesthésiste est de plus en plus requise à l'extérieur de la salle d'opération, en divers lieux de l'hôpital. Avec les développements rapides de la médecine, il y a eu une prolifération du nombre de procédures accomplies par des médecins non-chirurgiens. Celles-ci peuvent être inconfortables ou impliquer des complications sévères. Le rôle de l'anesthésiste devient donc important pour la sécurité et le confort du patient. Certaines de ces procédures pour lesquelles l'anesthésiste peut être requis sont énumérées dans le Tableau.

\section{Considérations générales}

On peut éliminer la réticence à administrer l'anesthésie dans les environnements moins familiers par une planification attentive, une bonne connaissance du patient, de l'intervention, de l'équipement disponible et des difficultés propres à l'environnement.'

\section{Le patient}

L'évaluation du patient est la même que pour toute intervention sous anesthésie en salle d'operation. Certaines interventions sont faites de routine sous anesthésie générale. Les raisons pour impliquer la présence d'un anesthésiste sont habituellement l'incapacité du patient à coopérer, l'inconfort propre à l'intervention, ou la présence d'un patient instable sur le plan médical. Les patients non-coopératifs nécessitent habituellement une anesthésie générale, et les autres patients peuvent être contrôlés avec neuroleptanesthésie ou une sédation légère, une surveillance étroite et de la réassurance.

\section{Interventions}

S'il veut être en mesure d'administrer une anesthésie adéquate et adaptée, l'anesthésiste doit connaître les détails de l'intervention, comment elle est exécutée, la douleur provoqué, sa durée et les complications possibles.

\section{Équipement}

L'équipement requis pour l'anésthesie générale doit être présent ou disponible immédiatement. On doit aussi appliquer les mêmes niveaux de surveillance que pour une anesthésie en salle d'opération. Il faut donc des moniteurs pour l'électrocardiographie, la tension artérielle, la température, la saturation d'oxygène et la mesure du $\mathrm{CO}_{2}$. Les médicaments, autant pour l'anesthésie générale que pour la réanimation, doivent être disponibles immédia-
TABLEAU Procédures à l'extérieur de la salle d'opération

Electrocorricothérapie (ECT)

Cardiologie

cardioversion

angiographie

insertion de pacemaker

insertion et vérification de defibrillateur

angioplastie

Radiologie

angiographie

tomographie axiale

résonnance magnétique nucléaire

cholangio-pancréatographie rétrograde par voie endoscopique

myélographie

rhizotomie percutanée

neuroradiologie d'intervention

Radiothérapie

Lithotripsie

tement ; si les substances anesthésiques sont entreposées dans un endroit éloigné, il faut s'assurer que les dates de péremption ne sont pas dépassées. Les accessoires tels que solutés, pièces buccales, laryngoscope, tubes endotrachéaux, stéthoscopes, appareils d'humidication et de réchauffement et sondes nasales doivent être amenés sur place. Un équipement de défibrillation et de réanimation doit être entreposé à proximité. Quelques centres ont un chariot de transport avec tout le nécessaire.

\section{Risques liés aux emplacements}

À l'extérieur de la salle d'opération, l'anesthésiste doit s'adapter à l'équipement nécessaire à l'intervention. Il peut y avoir des limites d'accès au patient, la possibilité d'exposition aux radiations, des salles sombres et froides, des tables inconfortables pour le patient et un environnement hostile pour la surveillance électronique. L'anesthésiste pourra être aussi seul dans cet environnement, il peut $n$ 'y avoir personne d'autre dans la salle qui comprendra les complexité de l'anesthésie. L'anesthésiste doit savoir où réclamer de l'assistance dans le cas d'une urgence. Une salle de réveil est l'endroit idéal pour la récupération du patient. Si elle est éloignée, il faut s'assurer que le patient est bien éveillé et stable avant d'en assurer le transport. 
La technique d'anesthésie variera selon l'intervention, l'emplacement et le besoin du patient. La conduite de l'anesthésie pour les interventions les plus fréquentes hors de la salle d'opération sera discutée ici et l'application de ces principes suggérera un modèle à partir duquel on pourra planifier une anesthésie à tout endroit.

\section{Électrochocs}

Malgré le fait que la thérapie aux électrochocs demeure controversée, elle est toujours une pratique courante. ${ }^{2-5}$ On pratique l'électrocorticothérapie dans des emplacements désignés d'hôpitaux psychiatriques et généraux, sous anesthésie générale. La crise de grand mal amenée par l'électrocorticothérapie produit des changements physiologiques importants. Les changements cardiovasculaires comprennent une stimulation parasympathique initiale qui résultera en bradycardie et hypotension. Quelques secondes plus tard, une stimulation sympathique importante se produit avec tachycardie, hypertension, et dysrythmie.

\section{Raisons qui nécessitent une anesthésie générale}

1) L'amnésie rétrograde survient après une convulsion adéquate, mais le besoin de musculo-relaxant pour prévenir les fractures nécessite une hypnose. 2) L'anesthésie va diminuer les séquelles physiologiques. 3) L'anxiété liée à la répétition des traitements est diminuée considérablement par l'anesthésie générale.

\section{Considérations anesthésiques}

Le patient devrait avoir une évaluation pré-anesthésique complete. Puisque l'augmentation de pression artérielle peut produire des lésions neurologiques ou cardiovasculaires, il faut s'assurer que les hypertendus ont un bon contrôle de leur pression artérielle avant de les amener à la thérapie par ECT. La plupart des patients seront traités par psychotropes tels que les tricycliques, le lithium ou les inhibiteurs de monoamine oxidase. L'habitude de cesser les inhibiteurs de la monoamine oxydase deux semaines avant l'administration de l'anesthésie a été remise en question. ${ }^{6}$ Le lithium peut interagir avec les barbituriques et prolonger le temps de récupération, et peut aussi prolonger l'action de la succinylcholine et du pancuronium. ${ }^{7,8}$

Les facteurs qui influencent le choix de l'anesthésie sont : 1) Le début d'action et le temps de recouvrement de l'anesthésie doivent être rapides avec le minimum d'effets secondaires. 2) Les substances ne devraient pas modifier la duré de la convulsion. 3) Comme la procédure se répète ces agents doivent être acceptables pour utilisation répétée.

\section{Anticholinergiques}

On n'a pas démontré de bénéfices à l'utilisation de routine d'atropine ou de glycopyrrolate en prémédication dans le but de diminuer la stimulation vagale et les sécrétions; cependant ils doivent être disponibles. ${ }^{9}$

\section{Agents d'induction}

Les substances les plus souvent utilisées sont le méthohexital et le thiopental. D'autres substances comprennent le diazepam, la kétamine, l'étomidate et plus récemment le propofol. Le diazepam augmente le seuil de convulsions. Le propofol va diminuer les effets cardiovasculaires et diminuer le temps de récupération. ${ }^{10}$ Cependant, le propofol peut réduire la durée de la convulsion et ainsi diminuer sa valeur thérapeutique. " La douleur produite à l'injection de méthohexital et de propofol sera réduite en utilisant de la lidocaine intra-veineuse. ${ }^{12}$

\section{Musculo-relaxants}

La succinylcholine est l'agent le plus populaire. La douleur musculaire n'est pas fréquente après l'utilisation de succinylcholine au cours d'électrocorticothérapie. ${ }^{13}$ Les agents non-dépolarisants ont été utilisés lorsque la succinylcholine était contre-indiquée. ${ }^{14}$

L'ajustement des doses et la synchronisation avec le choc demande une coopération entre l'anesthésiste et le psychiatre. Les dommages à la dentition, à la langue, aux yeux, aux extrémités et à la peau peuvent se produire si on n'utilise pas un protecteur buccal et des mesures de protection par le personnel présent. On pourra envisager l'utilisation de nitroprussiate, d'hydralazine, de clonidine ou de propranolol chez les patients avec maladie cardiovasculaire pour diminuer la réponse sympatique à l'ECT. ${ }^{15}$ Une préoxygenation et une ventilation manuelle avec oxygène jusqu'au retour de la ventilation spontanée diminueront l'incidence de dysrythmies. ${ }^{16}$ Un réanimateur de type ambu pourra être utilisé. Le patient devra récupérer sur le côté et être observé de près pour au moins 15 minutes après la convulsion, puisque cette période est à haut risque. ${ }^{2}$

\section{Cardioversion}

La cardioversion est utilisée pour ramener un rythme sinusal dans les dysrythmies cardiaques telles la fibrillation et le flutter auticulaire, la tachycardie supraventriculaire et ventriculaire. ${ }^{17}$ Le patient peut avoir développé cet épisode de façon aiguë avec une instabilité cardiovasculaire qui nécessite un traitement rapide, ou le problème peut être chronique avec absence de réponse à la thérapie usuelle, et dès lors le patient peut nécessiter une cardioversion élective. La cardioversion est habituellement exécutée dans les laboratoires d'hémodynamique, ou dans des unités avec surveillance électronique. Elle nécessite une anesthésie de courte durée pour éliminer la prise de conscience du choc électrique. Il n'est pas néces- 
saire de relâcher le patient puisqu'il y a seulement des contractures musculaires régionales. Les agents anesthésiques comprennent le thiopental, le méthohexital, le protoxyde d'azote, l'halothane, le diazépam, le midazolam et plus récemment le propofol. Le thiopental est le plus utilisé. Une benzodiaépine pourra être considérée comme l'agent de choix chez les patients à risque élevé ou dans les situations d'urgence, puisque les benzodiazépines produisent moins de dépression cardio-vasculaire. ${ }^{18} \mathrm{Ce}$ pendant, les patients peuvent avoir un souvenir de l'événement. Les avantages du midazolam sont sa plus courte durée d'action et la douleur réduite à l'injection. ${ }^{19}$ Le propofol produit un recouvrement rapide. ${ }^{20}$ La synchronisation entre l'anesthésiste et le cardiologue est essentielle. Le patient devrait être oxygéné et s'il y a dépression respiratoire, il faut le ventiler manuellement. Lorsque plus d'un choc doit être administré pour assurer la cardioversion, l'anesthésiste doit s'assurer que le patient demeure endormi.

\section{Interventions radiologiques}

Il y a plusieurs interventions diagnostiques et thérapeutiques en radiologie, où la sédation et la surveillance du patient sont habituellement assurés par le radiologiste. On demande à l'occasion la présence de l'anesthésiste pour s'occuper de patients agités, non coopératifs ou instables.

\section{Imagerie par résonnance magnétique}

Les difficultés de l'anesthésie des patients dans les salles de résonnance magnétique sont liées aux problèmes amenés par le champ magnétique. ${ }^{21,22}$ 1) La salle de résonnance magnétique est habituellement dans un endroit éloigné et isolé. 2) La chambre magnétique cylindrique de large dimension entoure le corps, ce qui limite l'accès au patient. 3) Les objets ferromagnétiques peuvent être attirés brutalement vers l'aimant ce qui en fait des projectiles dangeureux. 4) Les objets de métal de dimension importante peuvent modifier la qualité de l'image. 5) Plusieurs instruments électroniques ne fonctionneront pas de façon efficace à proximité de l'aimant. 6) Les objets ferreux implantables tels que des clips cérébrovasculaires, les clips chirurgicaux et les pace-makers présentent des risques.

\section{Équipement}

Les matériaux ferreux sont présents dans la plupart des equipements d'anesthésie tels que ics ventilateurs, vaporisateurs, cylindres, batteries de laryngoscopes, stéthoscopes, adapteurs, et les tiges de support pour infusion intra-veineuse. La plupart des équipements peuvent être modifiés ou construits à partir d'alliages non ferreux. La machine d'anesthésie représente le plus grand défi. Elle peut être fixée au mur dans la salle de résonnance magnétique ou être laissé à l'extérieur de la salle. Une autre solution est de dessiner ou modifier une machine d'anesthésie destinée à cette seule salle de résonnance magnétique. ${ }^{23}$ Des ventilateurs compatibles avec la résonnance magnétique sont disponibles. ${ }^{24} \mathrm{Si}$ les gaz ne sont pas disponibles en tuyauterie, les cylindres doivent être conservés à l'extérieur de la salle ou être remplacés par des cylindres d'aluminium.

\section{Moniteurs}

Il $\mathrm{y}$ a des modifications à apporter. ${ }^{25}$ De longs conduits sont essentiels pour tous les moniteurs et les lignes intraveineuses. On utilisera soit de l'électrocardiographie par télémétrie, avec filtre pour diminuer l'interférence magnétique, soit un doppler, ou un stéthoscope œsophagien précordial en plastique pour mesurer la fréquence cardiaque. Les tubulures rattachées au brassard de pression artérielle pourront être modifiés avant de les joindre à l'appareil de mesure non-invasive automatique. La ventilation sera surveillée par un «coussin » détecteur d'apnée, l'oxymétrie digitale et/ou la capnographie. S'il y a situation d'urgence, le champ magnétique peut être interrompu.

\section{Conduite de l'anesthésie}

Pour la tomographie avec résonance magnétique, la plupart des patients ont besoin d'une sédation légère ou de neurolept-analgésie mais un certain nombre auront besoin d'anesthésie générale. Quelques anesthésistes se font les tenants de l'intubation endotrachéale pour tous les patients qui ont besoin d'anesthésie générale. ${ }^{22}$ La ventilation spontanée peut être plus sécuritaire dans bien des situations, mais la condition médicale des patients peut obliger à la ventilation mécanique (patients de soins intensifs). La conduite à tenir et le choix des médicaments dépendra du patient et de l'équipement disponible.

\section{Neuroradiologie d'intervention}

Les techniques invasives de neuroradiologique telles l'embolisation ou l'occlusion par ballon de malformation artério-veineuse, de tumeur vasculaire, d'anévrysme intra-crânien et de fistule comportent on risque élevé de complications désastreuses. Celles-ci comprennent une hémorragie soudaine à la suite de rupture de lésions, l'ischémie cérébrale aiguë ou des problèmes techniques tels la migration de cathéter ou de ballon. Ces problèmes peuvent amener une détérioration rapide de l'état neurologique et/ou cardio-respiratoire du patient. Le patient peut dès lors nécessiter une ventilation rapide ou même une crâniotomie d'urgence. La surveillance de ces patients doit comprendre une évaluation continue de leur état neurologique. La méthode idéale est la neuroleptanalgésie qui permet la communication avec le patient. ${ }^{26}$ 


\section{Résumé}

L'administration de l'anesthésie aux patients à l'extérieur de la salle d'opération sera une expérience satisfaisante si l'on respecte les mêmes règles de soins que dans la salle d'opération et si l'on a planifié d'avance le soin du patient.

\section{Références}

(Voir page R128) 